\title{
Expansão do Sistema FreeFlow-3D para Escoamentos com Influência da Temperatura
}

M.H. SABATINI, A. CASTELO, N. MANGIAVACCHI, V.G. FERREIRA, M.F. TOMÉ, J.A. CUMINATO, Departamento de Ciências de Computação e Estatística, Instituto de Ciências Matemáticas e de Computação, ICMC-USP, 13560-970 São Carlos, SP, Brasil.

Resumo. O objetivo do presente trabalho é estender o ambiente de simulação FreeFlow-3D para a solução numérica de problemas de escoamentos incompressíveis, com superfícies livres e com influência de temperatura. Em conjunto com condições iniciais e de fronteira associadas, as equações de conservação são discretizadas no contexto de diferenças finitas. Os efeitos da temperatura são representados pela aproximação de Boussinesq, e a viscosidade é calculada em função da temperatura. Resultados numéricos, demostrando a capacidade do código FreeFlow-3D em resolver problemas tridimensionais não-isotérmicos e com superfícies livres, são apresentados.

\section{Introdução}

Com o contínuo avanço tecnológico dos computadores e das técnicas numéricas, é possível hoje a modelagem/simulação de problemas de grande porte em dinâmica dos fluidos. Nos últimos trinta anos, muitos problemas nessa área têm sido investigados, especialmente a classe dos problemas de escoamentos envolvendo superfícies livres.

Com base na metodologia SMAC, Tomé e McKee em 1994 desenvolveram o método GENSMAC [1], para a solução numérica de escoamentos de fluidos newtonianos, incompressíveis, com superfícies livres, e em geometrias bidimensionais arbitrárias. Mais tarde, Castelo e et al. [3] desenvolveram o sistema FreeFlow-3D, um ambiente integrado de modelagem, de simulação e de visualização para problemas de escoamentos transientes com superfícies livres e tridimensionais (3D). O sistema FreeFlow-3D adota a metodologia GENSMAC-3D [2] para a solução numérica das equações de conservação.

Este trabalho é uma extensão do sistema FreeFlow-3D, para a simulação de problemas de escoamentos incompressíveis com superfícies livres, em 3D e com influência da temperatura. Isto permitirá investigar a variação de temperatura e/ou a transferência de calor no fluido. 


\section{Equações de Conservação}

As equações de conservação que modelam problemas de escoamentos de fluidos newtonianos e incompressíveis são continuidade e Navier-Stokes. Em notação vetorial essas equações são, respectivamente, representadas por

$$
\begin{gathered}
\nabla \cdot \mathbf{u}=0, \\
\rho \frac{D \mathbf{u}}{D t}=\nabla \cdot \boldsymbol{\sigma}+\rho \mathbf{g},
\end{gathered}
$$

em que $\rho$ é a densidade, $\mathbf{u}(\mathbf{x}, t)$ a velocidade, $\boldsymbol{\sigma}$ o tensor de tensões e $\mathbf{g}$ a aceleração gravitacional. As equações constitutivas consideradas neste trabalho são

$$
\begin{gathered}
\boldsymbol{\sigma}=-p \mathbf{I}+\boldsymbol{\tau}, \\
\boldsymbol{\tau}=2 \mu(T) \mathbf{d}, \\
\mathbf{d}=\frac{1}{2}\left[(\nabla \mathbf{u})+(\nabla \mathbf{u})^{T}\right],
\end{gathered}
$$

em que $p=p(\mathbf{x}, t)$ é a pressão, $\mathbf{I}$ o tensor identidade, $\boldsymbol{\tau}$ o tensor extra tensão, $\mathbf{d}$ o tensor razão de deformação e $\mu=\mu(T)$ é a viscosidade em função da temperatura local T. Para a simulação de escoamentos com influência da temperatura utilizou-se neste trabalho um modelo matemático com base na aproximação de Boussinesq [5]. Nessa aproximação, todas as propriedades do fluido são consideradas constantes, exceto a densidade no termo fonte das equações de movimento que é assumida ter uma relação linear com a temperatura, isto é,

$$
\rho(T)=\rho_{\infty}+\left.\frac{\partial \rho}{\partial T}\right|_{T_{\infty}}\left(T-T_{\infty}\right)+O\left(T-T_{\infty}\right)^{2},
$$

que pode ser escrita como

$$
\rho(T) \approx \rho_{\infty}\left[1-\beta\left(T(\mathbf{x}, t)-T_{\infty}\right)\right]
$$

em que $\rho_{\infty}$ e $T_{\infty}$ são valores de referência de densidade e de temperatura, respectivamente, e $\beta=-\left.\frac{1}{\rho_{\infty}} \frac{\partial \rho}{\partial T}\right|_{T_{\infty}}$ o coeficiente de expansão térmica.

As equações de conservação de massa e de quantidade de movimento (2.1) e (2.2) podem ser, então, escritas em coordenadas cartesianas tridimensionais como

$$
\begin{aligned}
& \frac{\partial u}{\partial t}+\frac{\partial u^{2}}{\partial x}+\frac{\partial(u v)}{\partial y}+\frac{\partial(u w)}{\partial z}=-\frac{1}{\rho} \frac{\partial p}{\partial x}+\nu(T)\left(\frac{\partial^{2} u}{\partial x^{2}}+\frac{\partial^{2} u}{\partial y^{2}}+\frac{\partial^{2} u}{\partial z^{2}}\right) \\
& +\left[2 \frac{\partial u}{\partial x} \frac{\partial \nu(T)}{\partial x}+\left(\frac{\partial u}{\partial y}+\frac{\partial v}{\partial x}\right) \frac{\partial \nu(T)}{\partial y}+\left(\frac{\partial u}{\partial z}+\frac{\partial w}{\partial x}\right) \frac{\partial \nu(T)}{\partial z}\right] \\
& +\left[1-\beta\left(T(\mathbf{x}, t)-T_{\infty}\right)\right] g_{x},
\end{aligned}
$$




$$
\begin{aligned}
\frac{\partial v}{\partial t}+ & \frac{\partial(u v)}{\partial x}+\frac{\partial v^{2}}{\partial y}+\frac{\partial(v w)}{\partial z}=-\frac{1}{\rho} \frac{\partial p}{\partial y}+\nu(T)\left(\frac{\partial^{2} v}{\partial x^{2}}+\frac{\partial^{2} v}{\partial y^{2}}+\frac{\partial^{2} v}{\partial z^{2}}\right) \\
+ & {\left[\left(\frac{\partial u}{\partial y}+\frac{\partial v}{\partial x}\right) \frac{\partial \nu(T)}{\partial x}+2 \frac{\partial v}{\partial y} \frac{\partial \nu(T)}{\partial y}+\left(\frac{\partial w}{\partial y}+\frac{\partial v}{\partial z}\right) \frac{\partial \nu(T)}{\partial z}\right] } \\
+ & {\left[1-\beta\left(T(\mathbf{x}, t)-T_{\infty}\right)\right] g_{y}, } \\
\frac{\partial w}{\partial t}+ & \frac{\partial(u w)}{\partial x}+\frac{\partial(v w)}{\partial y}+\frac{\partial w^{2}}{\partial z}=-\frac{1}{\rho} \frac{\partial p}{\partial z}+\nu(T)\left(\frac{\partial^{2} w}{\partial x^{2}}+\frac{\partial^{2} w}{\partial y^{2}}+\frac{\partial^{2} w}{\partial z^{2}}\right) \\
+ & {\left[\left(\frac{\partial u}{\partial z}+\frac{\partial w}{\partial x}\right) \frac{\partial \nu(T)}{\partial x}+\left(\frac{\partial v}{\partial z}+\frac{\partial w}{\partial y}\right) \frac{\partial \nu(T)}{\partial y}+2 \frac{\partial w}{\partial z} \frac{\partial \nu(T)}{\partial z}\right] } \\
+ & {\left[1-\beta\left(T(\mathbf{x}, t)-T_{\infty}\right)\right] g_{z}, }
\end{aligned}
$$

em que $\left[1-\beta\left(T(\mathbf{x}, t)-T_{\infty}\right)\right] \mathbf{g}$ é o termo fonte. Para se determinar a convecção e a difusão da temperatura no domínio, deve-se resolver a equação da energia

$$
\frac{\partial T}{\partial t}+\frac{\partial(u T)}{\partial x}+\frac{\partial(v T)}{\partial y}+\frac{\partial(w T)}{\partial z}=\alpha\left(\frac{\partial^{2} T}{\partial x^{2}}+\frac{\partial^{2} T}{\partial y^{2}}+\frac{\partial^{2} T}{\partial z^{2}}\right),
$$

em que $\alpha=\frac{\kappa}{\rho c_{p}}$ é o coeficiente de difusividade térmica, sendo, respectivamente, $\kappa$ e $c_{p}$ o coeficiente de condutividade térmica e o calor específico à pressão constante. Portanto, as equações a serem resolvidas, para escoamentos com influência de temperatura e com a viscosidade variando, são as equações (2.8)-(2.11), mais a equação de conservação de massa (2.1).

Para adimensionalização das equações, definem-se as transformações

$$
\begin{gathered}
\mathbf{u}=U \mathbf{u}^{*}, \mathbf{x}=L \mathbf{x}^{*}, \nu(T)=\nu_{0} \nu^{*}(T), p=\rho_{\infty} U^{2} p^{*}, \rho=\rho^{*} \rho_{\infty}, \\
t=\frac{L}{U} t^{*}, \mathbf{g}=g \mathbf{g}^{*}, T=T^{*} \Delta T+T_{\infty}, \beta=\beta^{*} \Delta T,
\end{gathered}
$$

em que $U$ é uma velocidade característica e $L$ um comprimento característico. $\nu_{0}$ é uma viscosidade de referência, $\Delta T=T_{\max }-T_{\min }$ é a diferença entre as temperaturas máxima e mínima. $T_{\infty}$ é uma temperatura de referência, que é tomada como $T_{\text {min }}{ }^{1}$ e $\rho_{\infty}$ uma densidade de referência. Substituindo-se essas transformações nas equações de conservação e desconsiderando-se o símbolo “*” para simplificar, obtem-se as equações de conservação na forma adimensional

$$
\begin{aligned}
& \frac{\partial u}{\partial x}+\frac{\partial v}{\partial y}+\frac{\partial w}{\partial z}=0 \\
& \frac{\partial u}{\partial t}+\frac{\partial\left(u^{2}\right)}{\partial x}+\frac{\partial(u v)}{\partial y}+\frac{\partial(u w)}{\partial z}=-\frac{\partial p}{\partial x}+\frac{\nu(T)}{\operatorname{Re}}\left(\frac{\partial^{2} u}{\partial x^{2}}+\frac{\partial^{2} u}{\partial y^{2}}+\frac{\partial^{2} u}{\partial z^{2}}\right) \\
& +\frac{1}{\operatorname{Re}}\left[2 \frac{\partial u}{\partial x} \frac{\partial \nu(T)}{\partial x}+\left(\frac{\partial u}{\partial y}+\frac{\partial v}{\partial x}\right) \frac{\partial \nu(T)}{\partial y}+\left(\frac{\partial u}{\partial z}+\frac{\partial w}{\partial x}\right) \frac{\partial \nu(T)}{\partial z}\right]+\frac{1}{\text { Fr }^{2}} g_{x}, \\
& \frac{\partial v}{\partial t}+\frac{\partial(u v)}{\partial x}+\frac{\partial\left(v^{2}\right)}{\partial y}+\frac{\partial(v w)}{\partial z}=-\frac{\partial p}{\partial y}+\frac{\nu(T)}{\operatorname{Re}}\left(\frac{\partial^{2} v}{\partial x^{2}}+\frac{\partial^{2} v}{\partial y^{2}}+\frac{\partial^{2} v}{\partial z^{2}}\right) \\
& +\frac{1}{\operatorname{Re}}\left[\left(\frac{\partial u}{\partial y}+\frac{\partial v}{\partial x}\right) \frac{\partial \nu(T)}{\partial x}+2 \frac{\partial v}{\partial y} \frac{\partial \nu(T)}{\partial y}+\left(\frac{\partial w}{\partial y}+\frac{\partial v}{\partial z}\right) \frac{\partial \nu(T)}{\partial z}\right]+\frac{1}{\operatorname{Fr}^{2}} g_{y},
\end{aligned}
$$

${ }^{1}$ Quando se escolhe $T_{\infty}=T_{m i n}$, tem-se $T^{*} \in[0,1]$ 


$$
\begin{aligned}
& \frac{\partial w}{\partial t}+\frac{\partial(u w)}{\partial x}+\frac{\partial(v w)}{\partial y}+\frac{\partial\left(w^{2}\right)}{\partial z}=-\frac{\partial p}{\partial z}+\frac{\nu(T)}{\operatorname{Re}}\left(\frac{\partial^{2} w}{\partial x^{2}}+\frac{\partial^{2} w}{\partial y^{2}}+\frac{\partial^{2} w}{\partial z^{2}}\right) \\
& +\frac{1}{\operatorname{Re}}\left[\left(\frac{\partial u}{\partial z}+\frac{\partial w}{\partial x}\right) \frac{\partial \nu(T)}{\partial x}+\left(\frac{\partial v}{\partial z}+\frac{\partial w}{\partial y}\right) \frac{\partial \nu(T)}{\partial y}+2 \frac{\partial w}{\partial z} \frac{\partial \nu(T)}{\partial z}\right] \\
& +(1-\beta T) \frac{1}{\operatorname{Fr}^{2}} g_{z}, \\
& \quad \frac{\partial T}{\partial t}+\frac{\partial(u T)}{\partial x}+\frac{\partial(v T)}{\partial y}+\frac{\partial(w T)}{\partial z}=\frac{1}{\operatorname{RePr}}\left(\frac{\partial^{2} T}{\partial x^{2}}+\frac{\partial^{2} T}{\partial y^{2}}+\frac{\partial^{2} T}{\partial z^{2}}\right),
\end{aligned}
$$

em que $\operatorname{Re}=\frac{U L}{\nu}, \operatorname{Fr}=\frac{U}{\sqrt{L g}}$ e $\operatorname{Pr}=\frac{\nu}{\alpha}$ são, respectivamente, os números de Reynolds, de Froude, de Prandtl e de Nusselt.

\section{Modelagem da Viscosidade}

A viscosidade $\nu=\nu(T)$ é conhecida, representando a dependência do fluido em função da temperatura. Para o tratamento da viscosidade, neste trabalho utilizaram-se dois modelos: um para simulações com fluidos gasosos; e o outro para fluidos líquidos. No caso de fluídos gasosos foi escolhido o modelo

$$
\nu(T)=a T^{2}+b T+c
$$

e, no caso de fluidos líquidos, empregou-se

$$
\nu(T)=\frac{1}{a T^{2}+b T+c} .
$$

Em ambos os modelos apresentados acima, $a, b$ e $c$ são constantes a serem determinadas de forma a ajustar, pelo método dos mínimos quadrados, um conjunto de dados de viscosidade em função da temperatura. O modelo (3.1) melhor se ajustou aos dados para fluidos gasosos e o modelo (3.2) melhor se ajustou aos dados para fluidos líquidos (maiores detalhes podem ser encontrados em [8]). Na literatura, ver por exemplo em [5], são encontradas tabelas de propriedades físicas de fluidos contendo esses dados.

\section{Procedimento Computacional}

O procedimento computacional utilizado para o cálculo das velocidades, pressão e temperatura é baseado no método GENSMAC-3D [2]. Admite-se que, em um dado instante de tempo $t=t_{0}$, o campo de velocidades $\mathbf{u}\left(\mathbf{x}, t_{0}\right)$ é conhecido e as condições de fronteira para a velocidade e pressão são dadas. Admitem-se também que a temperatura $T\left(\mathbf{x}, t_{0}\right)$ e as suas condições de fronteiras são conhecidas. Os campos de velocidades e temperatura no tempo $t=t_{0}+\delta t$ são calculados como segue:

1. Seja $\tilde{p}\left(\mathbf{x}, t_{0}\right)$ um campo de pressão que satisfaça a condição de tensão normal correta sobre a superfície livre do fluido em $t=t_{0}$; 
2. Calcula-se o campo de velocidade intermediário $\tilde{\mathbf{u}}(\mathbf{x}, t)$ por

$$
\begin{aligned}
& \frac{\partial \tilde{u}}{\partial t}=-\frac{\partial\left(u^{2}\right)}{\partial x}-\frac{\partial(u v)}{\partial y}-\frac{\partial(u w)}{\partial z}-\frac{\partial \tilde{p}}{\partial x}+\frac{\nu(T)}{\operatorname{Re}}\left(\frac{\partial^{2} u}{\partial x^{2}}+\frac{\partial^{2} u}{\partial y^{2}}+\frac{\partial^{2} u}{\partial z^{2}}\right) \\
& +\frac{1}{\operatorname{Re}}\left[2 \frac{\partial u}{\partial x} \frac{\partial \nu(T)}{\partial x}+\left(\frac{\partial u}{\partial y}+\frac{\partial v}{\partial x}\right) \frac{\partial \nu(T)}{\partial y}+\left(\frac{\partial u}{\partial z}+\frac{\partial w}{\partial x}\right) \frac{\partial \nu(T)}{\partial z}\right]+\frac{1}{\operatorname{Fr}^{2}} g_{x} \\
& \frac{\partial \tilde{v}}{\partial t}=-\frac{\partial(u v)}{\partial x}-\frac{\partial\left(v^{2}\right)}{\partial y}-\frac{\partial(v w)}{\partial z}-\frac{\partial \tilde{p}}{\partial y}+\frac{\nu(T)}{\operatorname{Re}}\left(\frac{\partial^{2} v}{\partial x^{2}}+\frac{\partial^{2} v}{\partial y^{2}}+\frac{\partial^{2} v}{\partial z^{2}}\right) \\
& +\frac{1}{\operatorname{Re}}\left[\left(\frac{\partial u}{\partial y}+\frac{\partial v}{\partial x}\right) \frac{\partial \nu(T)}{\partial x}+2 \frac{\partial v}{\partial y} \frac{\partial \nu(T)}{\partial y}+\left(\frac{\partial w}{\partial y}+\frac{\partial v}{\partial z}\right) \frac{\partial \nu(T)}{\partial z}\right]+\frac{1}{\operatorname{Fr}^{2}} g_{y}, \\
& \frac{\partial \tilde{w}}{\partial t}=-\frac{\partial(u w)}{\partial x}-\frac{\partial(v w)}{\partial y}-\frac{\partial\left(w^{2}\right)}{\partial z}-\frac{\partial \tilde{p}}{\partial z}+\frac{\nu(T)}{\operatorname{Re}}\left(\frac{\partial^{2} w}{\partial x^{2}}+\frac{\partial^{2} w}{\partial y^{2}}+\frac{\partial^{2} w}{\partial z^{2}}\right) \\
& +\frac{1}{\operatorname{Re}}\left[\left(\frac{\partial u}{\partial z}+\frac{\partial w}{\partial x}\right) \frac{\partial \nu(T)}{\partial x}+\left(\frac{\partial v}{\partial z}+\frac{\partial w}{\partial y}\right) \frac{\partial \nu(T)}{\partial y}+2 \frac{\partial w}{\partial z} \frac{\partial \nu(T)}{\partial z}\right] \\
& +(1-\beta T) \frac{1}{\operatorname{Fr}^{2}} g_{z},
\end{aligned}
$$

com $\tilde{\mathbf{u}}\left(\mathbf{x}, t_{0}\right)=\mathbf{u}\left(\mathbf{x}, t_{0}\right)$, utilizando a condição de fronteira correta para $\mathbf{u}\left(\mathbf{x}, t_{0}\right)$. As equações (4.1)-(4.3) são resolvidas utilizando-se aproximações por diferenças finitas;

3. Resolve-se a equação de Poisson

$$
\nabla^{2} \psi(\mathbf{x}, t)=\nabla \cdot \tilde{\mathbf{u}}(\mathbf{x}, t)
$$

com as seguintes condições de contorno:

$$
\begin{gathered}
\frac{\partial \psi}{\partial \mathrm{n}}=0 \quad \text { sobre a fronteira rígida, } \\
\psi=0 \quad \text { sobre a superfície livre, }
\end{gathered}
$$

em que n é a direção normal ao contorno rígido. A discretização da equação de Poisson gera um sistema de equações lineares que é resolvido pelo método de gradientes conjugados;

4. Atualiza-se o campo de velocidades por

$$
\mathbf{u}(\mathbf{x}, t)=\tilde{\mathbf{u}}(\mathbf{x}, t)-\nabla \psi(\mathbf{x}, t) ;
$$

5. Calcula-se a nova pressão por

$$
p=\tilde{p}\left(\mathbf{x}, t_{0}\right)+\frac{\psi(\mathbf{x}, t)}{\delta t}
$$

em que $\delta t$ é o passo no tempo;

6. Determina-se $T(\mathbf{x}, t)$ pela equação da energia (2.16). 


\section{Condições de Contorno}

$\mathrm{Na}$ superfície livre do fluido, as condições de fronteira são obtidas impondo-se tensões normal e tangencial nulas nesse contorno. Na ausência de tensão superficial, estas condições podem ser representadas como [2]

$$
\mathbf{n} \cdot \boldsymbol{\sigma} \cdot \mathbf{n}=0, \quad \mathbf{m} 1 \cdot \boldsymbol{\sigma} \cdot \mathbf{n}=0, \quad \mathbf{m} \mathbf{2} \cdot \boldsymbol{\sigma} \cdot \mathbf{n}=0,
$$

em que $\mathbf{n}=\left(n_{x}, n_{y}, n_{z}\right)$ é o vetor normal unitário, $\mathbf{m} 1=\left(m 1_{x}, m 1_{y}, m 1_{z}\right)$ e $\mathbf{m} \mathbf{2}=$ $\left(m 2_{x}, m 2_{y}, m 2_{z}\right)$ são os vetores tangenciais à superfície. O tensor de tensões $\boldsymbol{\sigma}$ é dado por (2.3). Por representar melhor, fisicamente, as trocas de calor entre a superfície do fluido e o ambiente, utilizou-se para a temperatura a condição de contorno de Robin

$$
-\nabla T \cdot \mathbf{n}=\mathrm{Nu}\left(T-T_{A}\right),
$$

em que $T_{A}$ é uma temperatura de referência, tomada no ambiente. As condicões de não-escorregamento e injeção compõem as condições de contorno na superfície rígida e na seção de entrada, respectivamente. As velocidades tangencial e normal em relação à parede sólida são definidas iguais a zero. Para a temperatura utilizouse a condição adiabática (fluxo de calor nulo entre parede e fluido), ou a condição de Dirichlet.

\section{Discretização das Equações de Conservação}

A discretização das equações foi realizada empregando-se o método de diferenças finitas em malhas deslocadas [2]. A malha é uniformemente espaçada em cada direção, tendo as células comprimento $\delta x$, largura $\delta y$ e altura $\delta z$. As velocidades são definidas nas faces das células. A pressão, a temperatura e a função potencial $\psi$ são calculadas nos centros das células computacionais. A equação do movimento na direção $x$ é discretizada na posição $\left(i+\frac{1}{2}, j, k\right)$, na direção y na posição $(i, j+$ $\left.\frac{1}{2}, k\right)$ e na direção $z$ na posição $\left(i, j, k+\frac{1}{2}\right)$. As derivadas temporais são aproximadas utilizando-se diferenças progressivas, enquanto que o gradiente de pressão e as derivadas espaciais dos termos viscosos são discretizadas por diferenças centradas. Os termos convectivos são representados pelo esquema VONOS (Variable-OrderNon-Oscillatory-Scheme), um método "upwind" de alta ordem e limitado. Análise e aplicação desse esquema "upwind" podem ser encontradas em [7].

\section{Resultados Numéricos}

Os resultados publicados na literatura sobre escoamentos com influência da temperatura são, de modo geral, para escoamentos confinados por paredes aquecidas

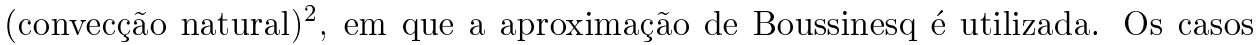
mais frequêntes são os problemas em que as paredes laterais são mantidas a uma

\footnotetext{
${ }^{2}$ Quando o campo de velocidades é determinado completamente pela temperatura, dá-se o nome de convecção natural [6].
} 
temperatura constante, enquanto as outras estão isoladas termicamente. Por essa razão, e pela dificuldade de se encontrar dados na literatura para problemas com superfícies livres, os resultados do modelo Boussinesq foram comparados com os apresentados em [6], para o caso da convecção natural no cubo fechado. O objetivo aqui é validar o simulador em problemas com influência da temperatura.

\subsection{Convecção Natural no Cubo Fechado}

Citado freqüentemente na literatura, o problema da convecção natural consiste em um cubo fechado com a temperatura mantida constante nas faces esquerda e direita, e as outras faces isoladas termicamente (ver Figura 1). Os dados para a si-

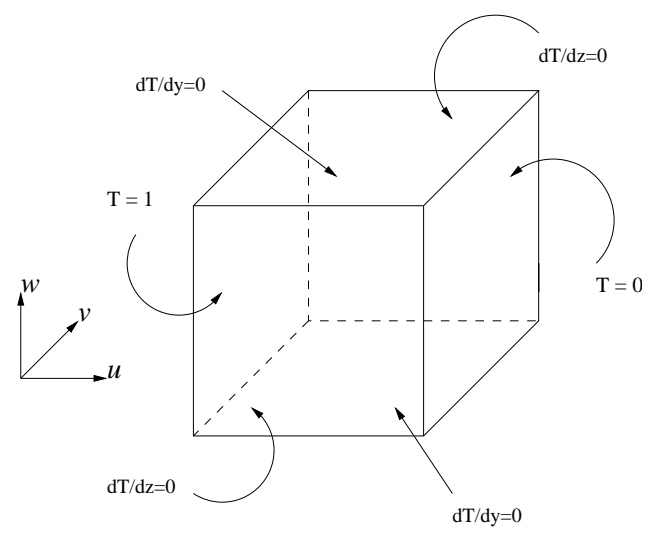

Figura 1: Modelo do problema da convecção natural.

mulação, extraídos de [4], foram: difusão $\alpha=2.08 \times 10^{-5} \frac{\mathrm{m}^{2}}{\mathrm{~s}}$; coeficiente de expansão $\beta=3.40 \times 10^{-3} K^{-1}$; viscosidade $\nu=1.5 \times 10^{-5} \frac{\mathrm{m}^{2}}{\mathrm{~s}}$; temperatura inicial do fluido $293 K$; parâmetros adimensionais $\operatorname{Re}=1.4, \operatorname{Pr}=0.72$. Os valores característicos foram $U=\frac{\alpha}{L}=0.00052 \frac{\mathrm{m}}{\mathrm{s}}$ e $L=0.04 \mathrm{~m}$. Este problema foi simulado em uma malha com $26 \times 26 \times 26$ células computacionais. A Tabela 1 compara os resultados numéricos obtidos com os resultados de [6].

\begin{tabular}{||l||c|r||r|r||r|r||}
\hline \hline \multicolumn{1}{||c||}{} & \multicolumn{2}{c||}{$R a=10^{3}$} & \multicolumn{2}{c||}{$R a=10^{4}$} & \multicolumn{2}{c||}{$R a=10^{5}$} \\
\cline { 2 - 7 } & SO & \multicolumn{1}{c||}{ Ref. [6] } & \multicolumn{1}{c|}{ SO } & \multicolumn{1}{c||}{ Ref. [6] } & \multicolumn{1}{c|}{ SO } & \multicolumn{1}{c|}{ Ref. [6] } \\
\hline \hline$u_{\max }$ & 3.521 & 3.543 & 17.327 & 16.719 & 45.908 & 43.900 \\
$v_{\max }$ & 0.143 & 0.173 & 2.348 & 2.156 & 8.477 & 9.690 \\
$w_{\max }$ & 3.630 & 3.544 & 20.040 & 18.983 & 70.396 & 71.060 \\
\hline \hline
\end{tabular}

Tabela 1: Resultados para Rayleigh $10^{3}, 10^{4}$ e $10^{5}$. SO: Solução Obtida.

Observa-se na Tabela 1 que pequenas diferenças em relação aos resultados de [6] são observadas. A seguir, na Figura 2, apresentam-se linhas de temperatura para 
o plano $y=0.5$ e números de Rayleigh $10^{3}, 10^{4}$ e $10^{5}$, que concordam satisfatoriamente com resultados da literatura (ver por exemplo [4]). Observa-se também que, com o aumento do número de Rayleigh, as isotérmas se aproximam da parede.

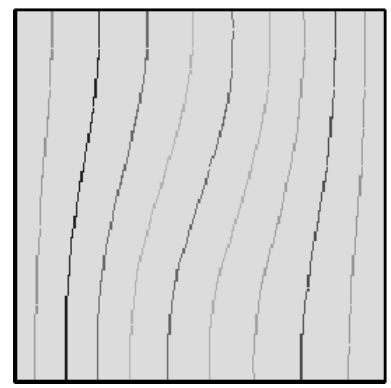

(a) $R a=10^{3}$

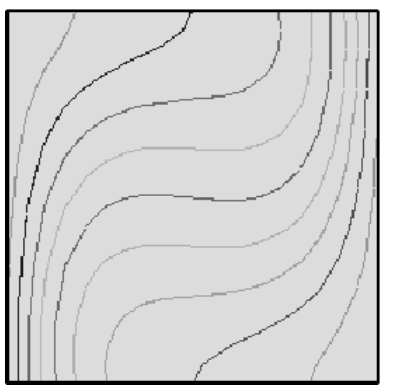

(b) $R a=10^{4}$

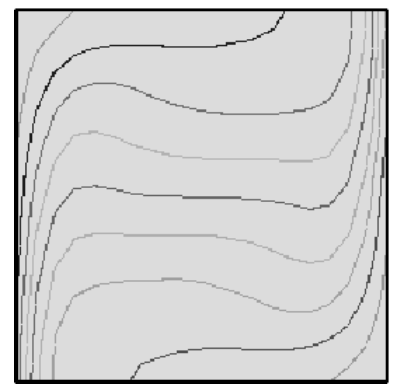

(c) $R a=10^{5}$

Figura 2: Linhas de Temperatura.
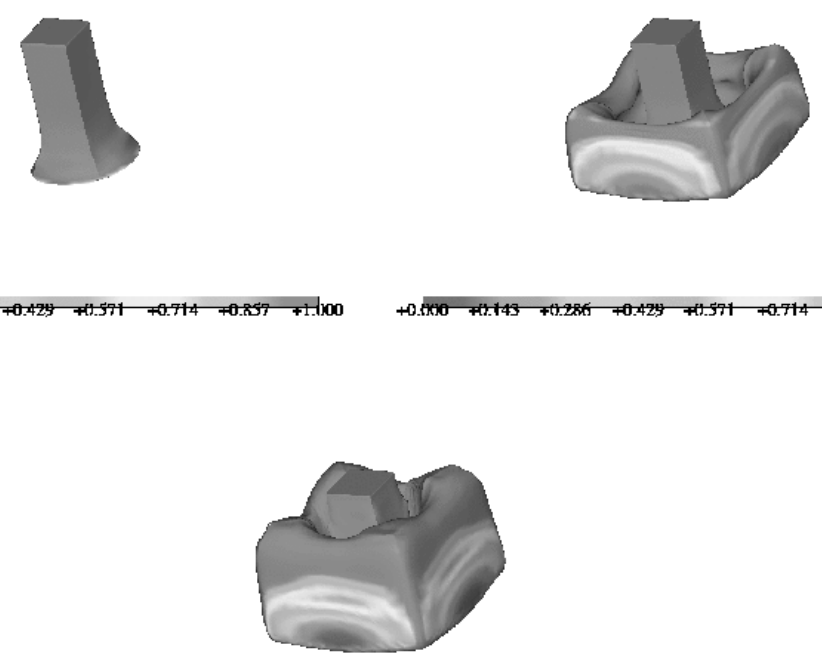

$+0.6100+1.143+0.286+0.429+0.571+0.744+0.257+1000$

Figura 3: Visualização da temperatura. 

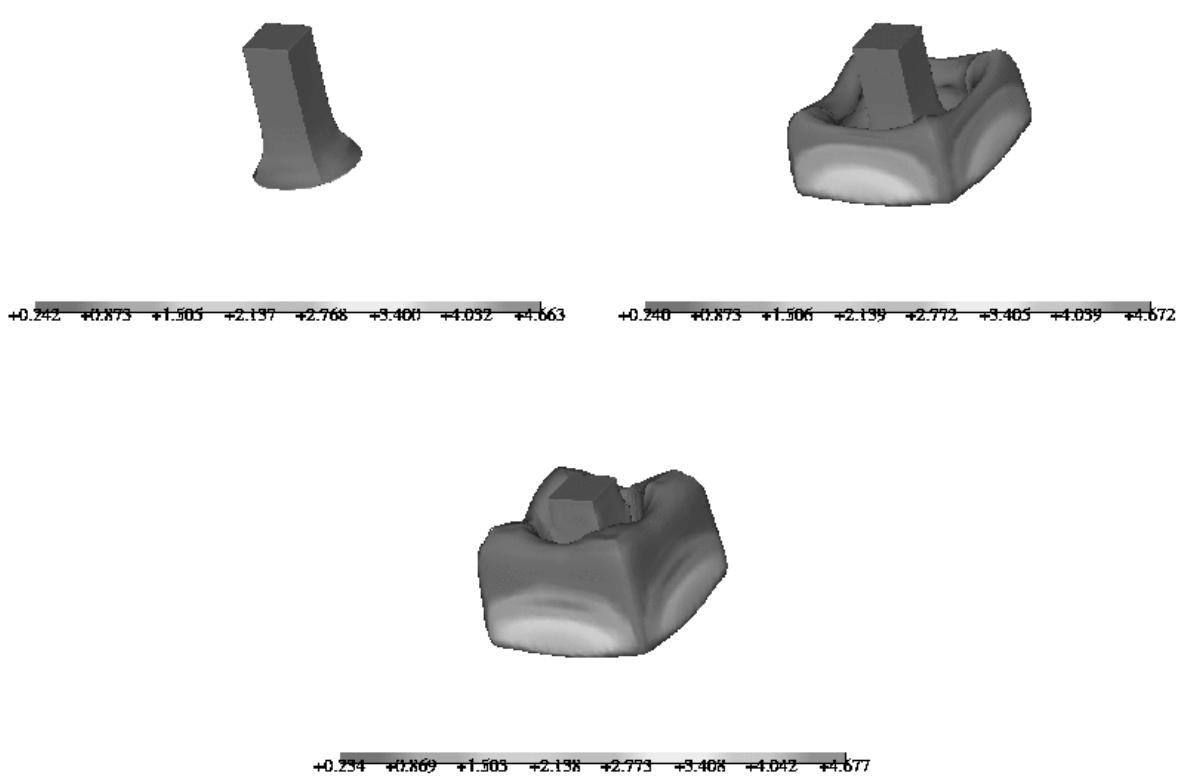

Figura 4: Visualização da viscosidade.

\subsection{Enchimento de um Recipiente}

Os objetivos desta simulação são: (i) mostrar um fluido, a uma dada temperatura, injetado em um recipiente com temperatura menor que a do fluido; e (ii) verificar o comportamento do escoamento ao entrar em contato com o recipiente. Para o cálculo da viscosidade, utilizou-se o modelo (3.2). As temperaturas do fluido (inicial), do ambiente e do recipiente foram, respectivamente, escolhidas como $1794.4 K, 1794.4 K$ e $1586.9 K$, sendo que a viscosidade do fluido cresceu a medida que a temperatura decresceu. Na Figura 3 observa-se o fluido sendo resfriado pelo recipiente, causando uma variação da viscosidade. Isto pode ser observado claramente na Figura 4.

\section{Conclusão}

Neste trabalho foi apresentado um modelo para a simulação de escoamentos 3D com superfícies livres, com influência da temperatura e com a viscosidade variando em função da temperatura. O modelo foi incorporado no sistema FreeFlow-3D. Os resultados numéricos ilustram a aplicabilidade do sistema FreeFlow-3D atual para escoamentos com superfície livre e com influência da temperatura. 


\begin{abstract}
The aim of this work is to extend the FreeFlow-3D simulation system to incompressible non-isothermal free-surface flows. Together with appropriate initial and boundary conditions, the conservation equations are discretized using the finite difference method. The temperature effects are included in the code by using the Boussinesq approximation, and the viscosity is calculated as a function of the temperature. Numerical results demonstrating the capabilities of the FreeFlow$3 \mathrm{D}$ code for solving tridimensional non-isothermal free-surface flows problems are presented.
\end{abstract}

\title{
Referências
}

[1] M.F. Tomé e S. McKee, GENSMAC: A Computational Marker-and-Cell Method for Free Surface Flows in General Domains, J. Comp. Phys., 110, No.1 (1994), 171-186.

[2] M.F. Tomé, A. Castelo Filho, J.A. Cuminato e S. McKee, GENSMAC-3D: A Numerical Method for Solving Three-Dimensional Free Surface Flows, International Journal of Numerical Method in Fluid, 37 (2001), 747-796.

[3] A. Castelo Filho, M.F. Tomé, C.N.L. César, S. McKee e J.A. Cuminato, FreFlow: An Integrated Simulation System for Three-Dimensional Free Surface Flows, Journal of Computing and Visualization in Science, 103 (1999), 77-92.

[4] J.M. Silva, "Simulação Numérica de Escoamentos com Superfícies Livres e com Influência da Temperatura", Dissertação de Mestrado, ICMC-USP, São Carlos, 2002.

[5] M. Griebel, D. Thomas e T. Neunhoeffer, "Numerical Simulation in Fluid Dynamics. A Practical Introduction", Society for Industrial and Applied Mathematics, Philadelphia, 1997.

[6] E. Trick, G. Labrosse e M. Betrouni, A First Incursion into the 3D Structure of Natural Convection of Air in a Differencially Heated Cubic Cavity, Internation Journal of Heat and Mass Transfer, 43 (2000), 4043-4056.

[7] V.G. Ferreira, M.F. Tomé, N. Mangiavacchi, A. Castelo Filho, J.A. Cuminato, A.O. Fortuna e S. McKee, High Order Upwinding and the hydraulic Jump, International Journal for Numerical Methods in Fluids, 39 (2002), 549-583.

[8] M.H. Sabatini, "Expansão do Sistema FreeFlow-3D para Escoamentos com Influência da Temperatura", Dissertação de Mestrado, ICMC-USP, São Carlos, 2002. 